AEI-2002-090

HUTP-02/A060

HU-EP-02/48

hep-th/yymmddd

\title{
Gravitational corrections in supersymmetric gauge theory and matrix models
}

\author{
Albrecht Klemm ${ }^{a}$, Marcos Mariño ${ }^{b}$ and Stefan Theisen ${ }^{c}$ \\ ${ }^{a}$ Humboldt Universität, Institut für Physik, Berlin, Germany \\ ${ }^{b}$ Jefferson Physical Laboratory, Harvard University, Cambridge, MA 02139, USA \\ ${ }^{c}$ Max-Planck-Institut für Gravitationsphysik, Albert-Einstein-Institut, Golm, Germany
}

\begin{abstract}
Gravitational corrections in $\mathcal{N}=1$ and $\mathcal{N}=2$ supersymmetric gauge theories are obtained from topological string amplitudes. We show how they are recovered in matrix model computations. This provides a test of the proposal by Dijkgraaf and Vafa beyond the planar limit. Both, matrix model and topological string theory, are used to check a conjecture of Nekrasov concerning these gravitational couplings in Seiberg-Witten theory. Our analysis is performed for those gauge theories which are related to the cubic matrix model, i.e. pure $S U(2)$ Seiberg-Witten theory and $\mathcal{N}=2 U(N)$ SYM broken to $\mathcal{N}=1$ via a cubic superpotential. We outline the computation of the topological amplitudes for the local Calabi-Yau manifolds which are relevant for these two cases.
\end{abstract}

November 2002 


\section{Introduction}

The fact that string theory can be a powerful tool to study four-dimensional supersymmetric gauge theories has been long appreciated. One of the most successful approaches is geometric engineering, where the gauge theory is appropriately embedded in a local Calabi-Yau compactification of type II string theory. A large and interesting class of supersymmetric field theories can be geometrically engineered in this way, including $\mathcal{N}=2$ supersymmetric Yang-Mills theory with or without matter [1] [2]. The gauge theory is recovered in a certain singular limit of the Calabi-Yau geometry where the string corrections decouple, and the gauge group and matter content of the field theory depend only on local properties of the Calabi-Yau space near the singularity.

Type II string theory on Calabi-Yau geometries has a topological subsector called topological string theory. It turns out that amplitudes in topological string theory compute exactly certain holomorphic terms in the effective action [3]. There are two types of topological strings, the A-model and the B-model. In the A-model, the relevant correlation functions are sums of world-sheet instanton contributions of a given genus. Using mirror symmetry one can relate the A-model to the B-model, and the latter provides an elegant and efficient tool to perform these sums exactly. From a pure field theory point of view the exact holomorphic information comes from summing over space-time instantons. The decoupling limit relates string world-sheet instantons to field theory space-time instantons, and for example the space-time instanton corrected exact gauge coupling of $\mathcal{N}=2$ Seiberg-Witten theory [4,5] is recovered in that limit from the genus zero topological string amplitude. Higher genus world-sheet instanton corrections are believed to calculate exactly, in the same limit, certain (holomorphic) couplings of the gauge theory to gravity. On the field theory side, these couplings haven't been much studied. For $\mathcal{N}=2$ theories, the genus one coupling can be extracted from the low-energy twisted theory, where the coupling to gravity plays an important role [6,7,8,9]. More recently there has been important progress in calculating also the space-time instantons contributions for higher genus [10,11.

In the context of geometric engineering of gauge theories, one can break $\mathcal{N}=2$ to $\mathcal{N}=1$ by adding space-time filling D-branes wrapped on internal supersymmetric cycles, or in a dual picture by turning on RR-fluxes. The best studied holomorphic $\mathcal{N}=1$ quantity is the superpotential, which is computed via genus zero open or closed topological string amplitudes, respectively. As in the $\mathcal{N}=2$ case, higher genus amplitudes give gravitational corrections to the gauge theory effective action [3, 12]. 
Recently Dijkgraaf and Vafa [13,14,15] have made the exciting proposal that these holomorphic terms in $\mathcal{N}=1$ and $\mathcal{N}=2$ supersymmetric field theories are calculated by matrix models. The genus expansion is replaced by an expansion in $1 / N, N$ being the dimension of the matrices. This leads to the possibility to use matrix models techniques to study supersymmetric gauge theories as well as topological string theories. In particular in the $\mathcal{N}=1$ context the tree level superpotential appears in the action of the matrix model and the exact effective low-energy superpotential is recovered in the planar limit. This has been checked explicitly in various examples, see for example [15, 116]. Although the original proposal of Dijkgraaf and Vafa applies to $\mathcal{N}=1$ models, one can recover results for the $\mathcal{N}=2$ theory in an appropriate limit [17, 15, [16].

The aim of this paper is to bring the above ideas together in order to perform a nontrivial check of the matrix model proposal beyond the planar limit, calculating thereby the corresponding gravitational terms in the supersymmetric field theories with completely independent methods. In this paper we will consider the simplest case of the cubic matrix potential. According to Dijkgraaf and Vafa, this matrix model captures the holomorphic couplings of IIB theory on a Calabi-Yau geometry with fluxes that generate a superpotential. On the other hand, $\mathcal{N}=2 S U(2)$ Seiberg-Witten theory is recovered at a special co-dimension one sub-locus in the moduli space, where the $\mathcal{N}=2$ supersymmetry is restored. This in turn can be geometrically engineered by decoupling the string correction from the dual type IIA string on the canonical line bundle over $\mathbb{P}^{1} \times \mathbb{P}^{1}=\mathbb{F}_{0}[\mathbb{1}$. The interrelations between these theories are shown in the diagram.

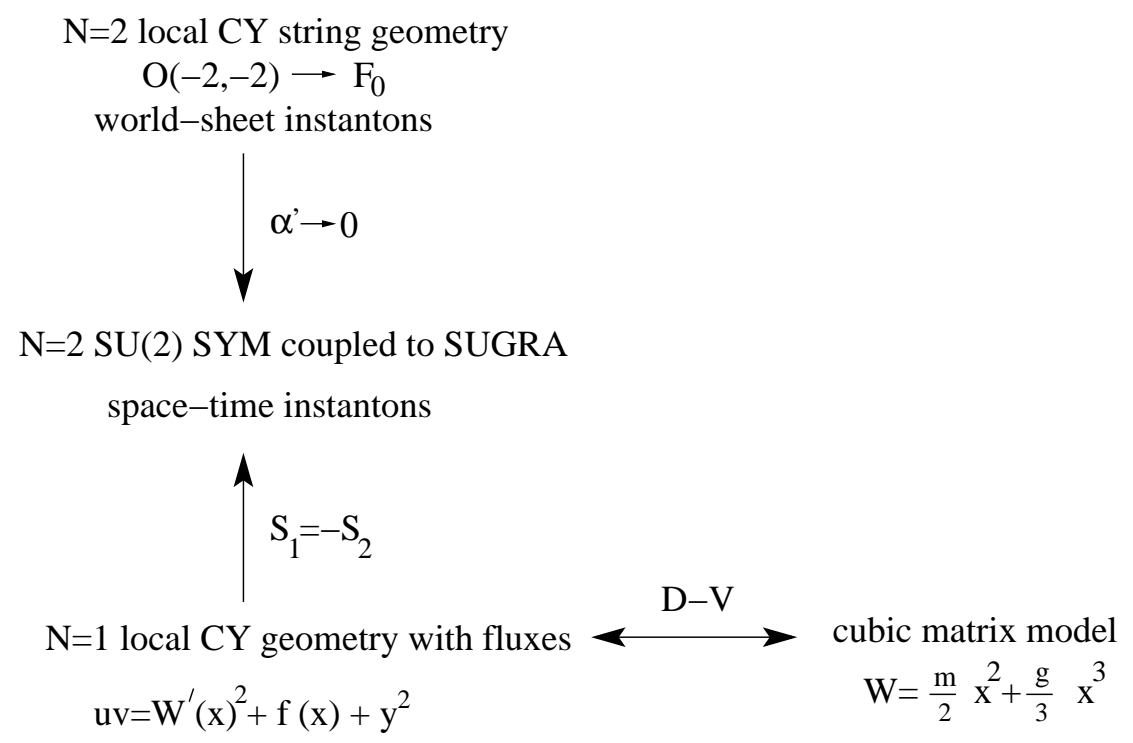


We are able to check non-planar contributions of the cubic matrix model against the holomorphic gravitational couplings in various ways. First, we calculate the exact genus one free-energy $F^{(1)}$ of the matrix model over the whole $\mathcal{N}=1$ moduli space. This matches the genus one contribution in the corresponding IIB model, which we obtained using the holomorphic anomaly equation. Second, we consider the holomorphic gravitational couplings of the $\mathcal{N}=2$ theory. We compute them in the string decoupling limit of type IIA theory on local $F_{0}$, and we check these in this limit against the corresponding space-time instanton calculations. Finally, we show that, by specializing the matrix model answer for $F^{(1)}$ to the $\mathcal{N}=2$ subspace, one recovers the right answer.

The paper is organized as follows. In section 2, we analyze topological string theory on the relevant local Calabi-Yau geometries: local $\mathbb{F}_{0}$, which geometrically engineers $\mathcal{N}=2$, $S U(2)$ Yang-Mills theory, and the geometries that engineer $\mathcal{N}=2 U(N)$ super Yang-Mills theory broken down to $\mathcal{N}=1$ by a tree level cubic superpotential $W(\Phi)$. In section 3, we derive an expression for the gravitational correction to $F^{(1)}$ of $\mathcal{N}=2, S U(2)$ Yang-Mills theory from topological field theory, and we show that it agrees with the decoupling limit of the corresponding amplitude on local $\mathbb{F}_{0}$. The geometries considered in section 2 are both captured by the cubic matrix model, which we analyze in section 4 from a perturbative point of view and also from the point of view of the loop equations. In particular, we derive an exact expression for $F^{(1)}$ that is shown to agree with the one computed in section 2 for the $\mathcal{N}=1$ theory. We also analyze the embedding of $\mathcal{N}=2$ in the matrix model and we reproduce the gravitational coupling of $S U(2)$ Seiberg-Witten theory from the genus one free energy of the matrix model. In section 5 we focus again on $S U(2), \mathcal{N}=2$ and we show that the results obtained in this paper agree with the direct instanton computations of [10,11], confirming in this way a conjecture of Nekrasov.

\section{Topological string in the associated local Calabi-Yau geometries}

In this section we will consider topological string theory on two different pairs of noncompact Calabi-Yau three-folds $I$ and $I I$. They are both related to the cubic matrix model in a way to be described below.

The first pair of Calabi-Yau three-folds, denoted by $I$, is a mirror pair. The A-model geometry of $I$ is the total space of the anti-canonical line bundle $\mathcal{O}(-2,-2) \rightarrow \mathbb{P}^{1} \times \mathbb{P}^{1}$. The B-model is its local mirror geometry, which is (in a patch) given by the constraint

$$
v w=1+Y_{1}+\frac{e^{-\hat{t}_{1}}}{Y_{1}}+Y_{2}+\frac{e^{-\hat{t}_{2}}}{Y_{2}}
$$


where $v, w \in \mathbb{C}$ and $Y_{1}, Y_{2} \in \mathbb{C}^{*} . \hat{t}_{i}$ are the two complex structure deformations. A canonical parameterization of the complex structure moduli space is in terms of periods $t_{i}$ of a meromorphic differential on the elliptic curve obtained from (2.1) by setting $v w=0$ [1]. The relation between $t_{i}$ and $\hat{t}_{i}$ will be given below. The Picard-Fuchs equations as well as explicit expressions for the periods in terms of various expansion parameters can be found in 1,18$]$.

The Calabi-Yau three-folds in the second pair, which will be denoted by $I I$ and $\widehat{I I}$, are related by a geometric transition. The $I I$ geometry is obtained by deforming the bundle $\mathcal{O}(-2) \oplus \mathcal{O}(0) \rightarrow \mathbb{P}^{1}$, such that the location of the $\mathbb{P}^{1}$ section in the $\mathcal{O}(0)$ direction, parameterized by $x$, is restricted to the two critical points, $a_{1}, a_{2}$, of a cubic potential $W(x)=\frac{m}{2} x^{2}+\frac{g}{3} x^{3}$ [19]. Consider now type IIB string theory on this Calabi-Yau threefold. As pointed out in [20,19], by wrapping $N_{1}, N_{2}$ D5 branes around the $\mathbb{P}_{1}$ 's located at $a_{1}, a_{2}$, respectively, one can geometrically engineer an $\mathcal{N}=1$ theory. This theory is $U(N) \mathcal{N}=2$ Yang-Mills theory broken to $\mathcal{N}=1$ via the addition of the superpotential $W(\Phi)$ for the $\mathcal{N}=1$ adjoint chiral superfield $\Phi$ that is part of the $\mathcal{N}=2$ gauge multiplet. The configuration with the above distribution of branes corresponds to a classical vacuum where $U(N) \rightarrow U\left(N_{1}\right) \times U\left(N_{2}\right)$. At low energies the $S U\left(N_{i}\right) \subset U\left(N_{i}\right)$ parts confine and the unbroken gauge group is a product of $U(1)$ factors. For each $S U\left(N_{i}\right)$ one has, at low energy, a glue-ball superfield $S_{i}$. The resulting dynamics is governed by the effective superpotential for the glue-balls, $W_{\text {eff }}\left(S_{i}\right)$.

The $\widehat{I I}$ geometry is obtained from $I I$ by a geometric transition [19], in which the two $\mathbb{P}^{1}$ 's are contracted to a point and the resulting singular space is smoothened by two $S^{3}$ 's of finite size. This has the local description as a hypersurface in $\mathbb{C}^{4}$ :

$$
v w=W^{\prime}(x)^{2}+f_{1}(x)+y^{2}
$$

where $x, y, v, w \in \mathbb{C}$ and $f_{1}$ is a polynomial of order one, which splits the double zeros of $W^{\prime}(x)^{2}$ to $a_{1}^{ \pm}, a_{2}^{ \pm}$. The two complex structure deformations of (2.2) are the two parameters of $f_{1}$ or, alternatively, the differences $a_{i}^{+}-a_{i}^{-}$. The periods $S_{i}=\frac{1}{2 \pi i} \int_{a_{i}^{-}}^{a_{i}^{+}} \omega$ and $\Pi_{i}=\frac{1}{2 \pi i} \int_{a_{i}^{+}}^{\Lambda} \omega, i=1,2$, where $\omega=\mathrm{d} x \sqrt{W^{\prime}(x)^{2}+f_{1}(x)}$, emerge by integrating over two dimensions of the period integrals of the holomorphic three-form of the local Calabi-Yau geometry (2.2) [19]. They are functions of the complex structure parameters and of the parameters $m$ and $g$ which appear in $W$. The $\Pi_{i}$ also depend on a cut-off $\Lambda$ which must be introduced since we are working on a non-compact curve (i.e. $x \in \mathbb{C}$ rather than $x \in \mathbb{P}^{1}$; otherwise there would be e.g. a linear relation $S_{1}+S_{2}=0$ ). After the geometric transition the D5 branes disappear, and we are left with a closed IIB string geometry with fluxes. 


\subsection{Topological amplitudes on $\mathcal{O}(-2,-2) \rightarrow \mathbb{P}^{1} \times \mathbb{P}^{1}$ and their field theory interpretation.}

The geometry $I$ was considered in [1] to geometrically engineer $S U(2)$ Seiberg-Witten field theory from string theory. The two moduli of the A-model are the complexified Kähler parameters $t_{1}$ and $t_{2}$ of the two $\mathbb{P}^{1}$ 's. String corrections to the gauge theory can be decoupled by sending $\epsilon \sim \sqrt{\alpha^{\prime}} \rightarrow 0$. This should be done in such a way as to preserve the renormalization group relation $t_{1} \sim 1 / g_{s}^{2} \sim 1 / g_{\mathrm{YM}}^{2} \sim \log \left(\frac{m_{W}}{\Lambda}\right)$ where $m_{W} \sim a \sim t_{2}, \Lambda$ is the scale of the gauge theory, $g_{s}$ the string coupling and $a$ parameterizes the expectation value of the adjoint scalar field in the Cartan subalgebra of $S U(2)$. This leads to the double scaling limit $1 \epsilon \rightarrow 0$ [21, []

$$
\begin{aligned}
\exp \left(-\frac{1}{g_{s}^{2}}\right) & =\exp \left(-t_{1}\right)=c_{1} \epsilon^{4} \Lambda^{4} \\
t_{2} & =\epsilon c_{2} a
\end{aligned}
$$

If we choose $c_{1}=\frac{1}{2}$ and $c_{2}=2, \Lambda$ and $a$ turn out to be those of the Seiberg-Witten theory. We will make this choice in the following.

Our aim is to solve the topological $B$-model on this geometry at higher genus and to take the field theory limit (2.3) of these contributions. In sect. 3 we will derive the genus one result directly as the holomorphic coupling of the $\mathcal{N}=2$ chiral multiplet to gravity and in sect. 4 we will recover it from non-planar contributions to the free energy of the cubic matrix model, evaluated at the minimum of the effective superpotential. It has been shown explicitly in [16] that the effective superpotential is computed by the planar diagrams. Our results thus provide a test of the Dijkgraaf-Vafa conjecture beyond the planar approximation.

The leading structure of the period vector in the large complex structure variables near $z_{i} \equiv e^{-\hat{t}_{i}}=0$ 目 is

$$
\Pi=\left(1, t_{1}=\log \left(z_{1}\right)+\sigma, t_{2}=\log \left(z_{2}\right)+\sigma, F_{q}=t_{1} t_{2}+\rho\right)^{t},
$$

where $\sigma=2\left(z_{1}+z_{2}\right)+3\left(z_{1}^{2}+4 z_{1} z_{2}+z_{2}^{2}\right)+O\left(z^{3}\right)$ and $\rho=4\left(z_{1}+z_{2}\right)+\left(9 z_{1}^{2}+32 z_{1} z_{2}+\right.$ $\left.9 z_{2}^{2}\right)+O\left(z^{3}\right)$. The formulae in [22] give the whole expansions. We denote $q_{i}=\exp \left(-t_{i}\right)$. With $q=q_{1} / q_{2}$ and $Q=q_{2}$ we can obtain the genus zero prepotential by integrating $F_{q}=-2 q \frac{\partial}{\partial q} F^{(0)}(q, Q)$, cf. [22]. For later use we define $Q=: e^{-T}$ and $q=: e^{-t}$.

1 In the heterotic-type II duality $t_{1}$ is identified with the size of the base of the K3 fibration and $t_{2}$ with the size of a 2-cycle in the fiber.

2 A schematic view of the moduli space can be found in 18 . 
The topological amplitudes $F^{(g)}$ for $g>0$ are not holomorphic in the complex structure parameters, but various holomorphic limits exist. For example, if we expand them around the large complex structure point where $q_{i} \rightarrow 0$ one can take the holomorphic limit $\bar{q}_{i} \rightarrow 0$ 23] in which $F^{(g)}$ has the form

$$
F^{(g)}=C^{(g)}+\sum_{\substack{m, n \geq 0 \\(m, n) \neq(0,0}}^{\infty} c_{n m}^{(g)} q_{1}^{n} q_{2}^{m}
$$

where $C^{(g)}$ stands for classical terms. The expansion parameters $c_{n m}^{(g)}$ are the GromovWitten invariants for the maps of genus $g$ world-sheets with bidegree $(m, n)$, while the classical terms arise from maps of bidegree $(0,0)$. In our case we have $C^{(0)}=\frac{1}{24} t_{1}^{3}-\frac{1}{8} t_{1}^{2} t_{2}-$ $\frac{1}{8} t_{1} t_{2}^{2}+\frac{1}{24} t_{2}^{3}, C^{(1)}=-\frac{t_{1}}{24}-\frac{t_{2}}{24}$ and $C^{(g)}=(-1)^{g} \frac{\left|B_{2 g} B_{2 g-1}\right|}{4 g(2 g-1)(2 g-1) !} \chi(\chi$ is the 'regularized' Euler number of the non-compact target space).

In the decoupling limit (2.3) the topological amplitudes $F^{(g)}$ compute the holomorphic part of the low-energy effective $\mathcal{N}=2$ SUSY field theory. In particular the complexified effective gauge coupling $\left(\tau=\frac{4 \pi i}{g^{2}}+\frac{\theta}{2 \pi}\right)$ is obtained in the field theory limit as $\tau(u)=$ $\frac{\partial^{2}}{\partial a^{2}} \mathcal{F}^{(0)}=\lim _{\epsilon \rightarrow 0} 4 \partial_{t_{2}}^{2} F^{(0)}$ [1], where the $\log (\epsilon)$ terms, which appear in this limit, have been absorbed in the bare coupling and $u(a)$ parameterizes the field theory moduli space. Similarly, the holomorphic functions $\mathcal{F}^{(g)}(u(a))$, which multiply the following combinations of the self-dual part of the graviphoton field strength, $F_{+}$, and of the curvature tensor, $R_{+}$, in the effective Lagrangian

$$
\mathcal{F}^{(g)}(u(a)) F_{+}^{2 g-2} R_{+}^{2}
$$

can be calculated from $F^{(g)}$ in the limit (2.3). On dimensional grounds, these terms are suppressed by $M_{p l}^{(2-2 g)} \sim \epsilon^{(2 g-2)}$.

A non-vanishing $n$-space-time-instanton contribution to $\mathcal{F}^{(g)}(u)=\lim _{\epsilon \rightarrow 0} \epsilon^{(2 g-2)} F^{(g)}$ arises from the infinite number of terms in $F^{(g)}$ at fixed degree $n$ (i.e. w.r.t. the base) in (2.5). This contribution must have the structure $a^{2-2 g}\left(\frac{\Lambda^{4}}{a^{4}}\right)^{n}$. This requires that the corresponding term in $f^{(g)}$ scales like $\frac{1}{(a \epsilon)^{2 g-2}}\left(\frac{\Lambda^{4}}{a^{4}}\right)^{n} \sim \frac{q_{1}^{4 n}}{\left(1-q_{2}\right)^{4 n+2 g-2}}$. This in turn implies that $c_{n m}^{(g)}$ grows with $m$ as

$$
\hat{c}_{n m}^{(g)}=\gamma_{n} m^{4 n-3+2 g} .
$$

A similar argument was given in [1] for $g=0$. The proportionality factor $\gamma_{n}$ is directly related to the gauge theory space-time instantons. E.g. for the gauge coupling we have

$$
\hat{c}_{n m}^{(0)}=\frac{2^{(4 n-1)}}{(4 n-3) ! n} \mathcal{F}_{n} m^{4 n-3},
$$


with $\mathcal{F}_{n}$ the $n$-instanton contribution to $\mathcal{F}^{(0)}$ as calculated in [24].

The topological amplitudes for this model in the large volume limit have been calculated for $g=0$ in [1] and for $g=1$ in [22]. Generally, the amplitudes $F^{(g)}$ for $g>1$ are defined recursively in terms of all amplitudes with lower $g$ and the propagators $S^{i j}$ as defined in [3]. It can be shown that for this model the propagators can be chosen to vanish except for $S^{t t}[18$. It can be determined from the $g=0,1$ amplitudes by using the simplification of the holomorphic anomaly equation which occurs at genus one in the case with only one non-zero propagator:

$$
\partial_{t} F^{(1)}=S^{t t} \partial_{t}^{3} F^{(0)}+\partial_{t} \sum_{r} a_{r} \log \left(\Delta_{r}\right)
$$

Here the sum is over the components of the discriminant loci which are (i) are the conifold locus $\Delta_{1}=1-8\left(z_{1}+z_{2}\right)+16\left(z_{1}^{2}+z_{2}^{2}\right)-32 z_{1} z_{2}$ and (ii) the divisors $\Delta_{2}=z_{1} z_{2}$ at large radius limit. The coefficients $a_{r}$ parameterize the holomorphic ambiguity. A convenient choice is $a_{1}=-\frac{1}{12}$ and $a_{2}=\frac{1}{12}$ which yields, using the genus 0,1 results, the expansion of $S^{t t}$

$$
S^{t t}=\frac{1}{2}+4 q_{1} q_{2}+16\left(q_{1}^{2} q_{2}+q_{1} q_{2}^{2}\right)+40\left(q_{1}^{3} q_{2}+q_{1} q_{2}^{3}\right)+188 q_{1}^{2} q_{2}^{2}+\mathcal{O}\left(q^{5}\right)
$$

With the recursive definition of the $F^{(g)}$ [3], worked out for the local $B$-model in [25], the higher $F^{(g)}$ can be calculated up to the holomorphic ambiguity. We have fixed the latter from the knowledge of the absence of holomorphic curves of low degree 26.

We have calculated the Gromov-Witten invariants using the above procedure and found agreement with genus 2 results of [27], who used the same method and at higher genus with the ones evaluated in [28] using Chern-Simons theory. In particular we find that the $c_{n m}^{(g)}$ are

$$
c_{n m}^{(g)}=P_{n}^{(4 n-3+2 g)}(m)=p_{n}^{(2 n-2+2 g)}(x) \prod_{k=1}^{2 n-1}(k+m)
$$

where $P_{n}^{(d)}(x), p_{n}^{(d)}(x)$ are polynomials of degree $d$. The remarkable fact that the GromovWitten invariants lie on polynomials is a consequence of the embedding of the $S U(2)$ $\mathcal{N}=2$ supersymmetric gauge theory in the string geometry and holds therefore also for other geometries like $\mathcal{O}(-K) \rightarrow \mathbb{F}_{n}$ with $n=1,2$, which allow for such an embedding [1]. 
We can give the topological string amplitudes exactly to all orders in $q_{2}$ by specifying the $P_{n}^{(2 n-2+2 g)}(x)$ or alternatively by writing

$$
\begin{aligned}
& F^{(0)}=C^{(0)}(t)-2\left(\operatorname{Li}_{3}\left(q_{2}\right)+\sum_{n=1}^{\infty} \frac{q_{1}^{n}}{n^{3}\left(1-q_{2}\right)^{4 n-2}} h_{n}^{(0)}\left(q_{2}\right)\right) \\
& F^{(1)}=C^{(1)}(t)-\frac{1}{6}\left(\operatorname{Li}_{1}\left(q_{2}\right)-\sum_{n=1}^{\infty} \frac{q_{1}^{n}}{n\left(1-q_{2}\right)^{4 n}} h_{n}^{(1)}\left(q_{2}\right)\right) \\
& F^{(g)}=C^{(g)}+\frac{(-1)^{g} B_{2 g}}{g(2 g-2) !} \sum_{n=0}^{\infty} \frac{q_{1}^{n}}{n^{3-2 g}\left(1-q_{2}\right)^{4 n+2 g-2}} h_{n}^{(g)}\left(q_{2}\right) .
\end{aligned}
$$

The $h_{n}^{(g)}$ are polynomials of degree $2(n+g-1)$ whose symmetric coefficients determine all orders in $q_{2}$ for given a order in $q_{1}$. E.g. for $g=0$ we find

$$
\begin{aligned}
& h_{1}^{(0)}=1 \\
& h_{2}^{(0)}=1+18 q+q^{2} \\
& h_{3}^{(0)}=1+98 q+450 q^{2}+98 q^{3}+q^{4} \\
& h_{4}^{(0)}=1+306 q+4851 q^{2}+13188 q^{3}+4851 q^{4}+\ldots \\
& h_{5}^{(0)}=1+732 q+26903 q^{2}+206434 q^{3}+426060 q^{4}+\ldots \\
& h_{6}^{(0)}=1+1490 q+105315 q^{2}+1660604 q^{3}+8358292 q^{4}+14651604 q^{5}+\ldots
\end{aligned}
$$

The missing terms, indicated by ..., can be recovered from the ones displayed via $h_{n}^{(g)}(1 / q)=q^{-(2(n+g-1)} h^{(g)}(q)$. (2.13) and (2.3) allow to determine the $\mathcal{F}_{n}$ and also provides an expansion scheme for the string corrections. E.g. using $c_{1}=\frac{1}{2}$ and $c_{2}=2$ we expand $F^{(0)}$ in the limit (2.3), using $\operatorname{Li}_{3}(1-\epsilon a)=\zeta(3)-\frac{\pi^{2}}{6} a \epsilon+\left(\frac{3}{4}-\frac{\pi^{2}}{12}-\frac{1}{2} \log (\epsilon a)\right) a^{2} \epsilon^{2}+\mathcal{O}\left(\epsilon^{3}\right)$. This yields to the relevant order, namely $\epsilon^{2}$, from (2.12) and (2.13)

$\mathcal{F}^{(0)}=a^{2}\left(\log (x)+c^{(0)}-\frac{x}{2^{5}}-\frac{5 x^{2}}{2^{14}}-\frac{3 x^{3}}{2^{18}}-\frac{1469 x^{4}}{2^{31}}-\frac{4471 x^{5}}{5 \cdot 2^{34}}-\frac{40397 x^{6}}{2^{43}}-\frac{441325 x^{7}}{7 \cdot 2^{47}}+\mathcal{O}\left(x^{8}\right)\right)$

where $x=\left(\frac{\Lambda}{a}\right)^{4}$ and $c^{(0)}$ is related to the bare gauge coupling into which a $\log (\epsilon)$ term has been absorbed. This matches the Seiberg-Witten prepotential [24]. 
The corresponding expressions for genus one are

$h_{1}^{(1)}=(1-q)^{2}$

$h_{2}^{(1)}=1-2 q-94 q^{2}-2 q^{3}+q^{4}$

$h_{3}^{(1)}=1-1137 q^{2}-3872 q^{3}-\ldots$

$h_{4}^{(1)}=1+4 q-6818 q^{2}-72168 q^{3}-158262 q^{4}-\ldots$

$h_{5}^{(1)}=1+10 q-28440 q^{2}-643440 q^{3}-3622665 q^{4}-6479092 q^{5}-\ldots$

$h_{6}^{(1)}=1+18 q-94008 q^{2}-3827252 q^{3}-41834673 q^{4}-167100606 q^{5}-265697392 q^{6}-\ldots$

The holomorphic quantities of the gauge theory from higher $\mathcal{F}^{(g)}$ 2.6) can be extracted in the limit (2.3) at order $\epsilon^{2-2 g}$, as explained above. For $\mathcal{F}^{(1)}$ we obtain from (2.12) and (2.15)

$\mathcal{F}^{(1)}=-\frac{1}{24} \log (x)+c^{(1)}+\frac{x^{2}}{2^{13}}+\frac{x^{3}}{3 \cdot 2^{14}}+\frac{1647 x^{4}}{2^{29}}+\frac{981 x^{5}}{2^{31}}+\frac{450137 x^{6}}{3 \cdot 2^{41}}+\frac{45111 x^{7}}{2^{42}}+\mathcal{O}\left(x^{8}\right)$,

this expression matches exactly the one calculated in the twisted $\mathcal{N}=2$ gauge theory in the next section and the matrix model calculation in section 4 .

We have calculated the $h_{i}^{(g)}$ with the topological B-model methods described in [18], albeit at another expansion point, up to genus 3. These expressions can be found in appendix A. In the gauge theory limit we get the following expressions for the holomorphic functions in (2.6)

$$
\begin{aligned}
& \mathcal{F}^{(2)}=\frac{1}{a^{2}}\left(-\frac{1}{480}-\frac{11 x^{2}}{2^{18}}-\frac{117 x^{3}}{2^{22}}-\frac{171201 x^{4}}{2^{34}}-\frac{1919923 x^{5}}{5 \cdot 2^{37}}-\frac{96877135 x^{6}}{2^{47}}+\mathcal{O}\left(x^{7}\right)\right) \\
& \mathcal{F}^{(3)}=\frac{1}{a^{4}}\left(-\frac{1}{8064}+\frac{7 x^{2}}{2^{19}}+\frac{293 x^{3}}{2^{23}}+\frac{985823 x^{4}}{2^{35}}+\frac{4069345 x^{5}}{5 \cdot 2^{38}}+\frac{416333277 x^{6}}{2^{46}}+\mathcal{O}\left(x^{7}\right)\right) .
\end{aligned}
$$

\subsection{The B-model on the local geometry $\widehat{I I}$}

As explained before, the geometry $\widehat{I I}$ describes the low-energy dynamics of $U(N)$ super Yang-Mills theory with a cubic tree-level superpotential, and Dijkgraaf and Vafa conjectured in 13] that the amplitudes of the topological B-model on this geometry, which correspond to the holomorphic couplings of the gauge theory, is captured by the cubic matrix model. At genus zero this was checked by noticing that the planar solution of the 
matrix model reproduces the special geometry of $\widehat{I I}$ [13], and it was also confirmed in [16] by using matrix model perturbation theory. To check this at genus one, we shall calculate $F^{(1)}$. As explained in [23,3], $F^{(1)}$ is a section of a determinant line bundle over the complex moduli space, which was studied by Ray and Singer. $F^{(1)}$ satisfies a holomorphic anomaly equation which can be readily integrated up to a holomorphic ambiguity to be discussed below.

To proceed we need the periods $S_{1}, S_{2}$ from [19], whose notation we adopt. It is convenient to change variables $\left(a_{1}^{-}, a_{1}^{+}, a_{2}^{-}, a_{2}^{+}\right) \equiv\left(x_{1}, x_{2}, x_{3}, x_{4}\right) \rightarrow\left(\Delta_{21}, \Delta_{43}, Q, I\right)$ where

$$
\begin{aligned}
\Delta_{21} & \equiv \frac{1}{2}\left(x_{2}-x_{1}\right), \quad \Delta_{43} \equiv \frac{1}{2}\left(x_{4}-x_{3}\right) \\
Q & \equiv \frac{1}{2}\left(x_{1}+x_{2}+x_{3}+x_{4}\right)=-\frac{m}{g} \\
I & \equiv \frac{1}{2}\left[\left(x_{3}+x_{4}\right)-\left(x_{1}+x_{2}\right)\right]=\sqrt{\Delta^{2}-2\left(\Delta_{21}^{2}+\Delta_{43}^{2}\right)}
\end{aligned}
$$

We will also use $z_{1}=\Delta_{43}^{2}$ and $z_{2}=\Delta_{21}^{2}$ and $\Delta=\left(a_{1}-a_{2}\right)=\frac{m}{g}$.

The periods $S_{i}\left(z_{1}, z_{2}, g, \Delta\right)$ were computed in $[19]$. To express the $B$-model amplitudes in terms of the $S_{i}$ we need the inverse relations

$$
\begin{aligned}
& z_{1}\left(S_{1}, S_{2}, g, \Delta\right)=\frac{4}{g \Delta} S_{1}+\frac{8}{g^{2} \Delta^{4}}\left(2 S_{1}^{2}-3 S_{1} S_{2}\right)+\frac{8}{g^{3} \Delta^{7}} S_{1}\left(5 S_{1}-13 S_{2}\right)\left(4 S_{1}-3 S_{2}\right)+\mathcal{O}\left(S^{4}\right) \\
& z_{2}\left(S_{1}, S_{2}, g, \Delta\right)=z_{1}\left(S_{2}, S_{1}, g,-\Delta\right)
\end{aligned}
$$

According to [23] and [3] and taking the simplification in the local case [25] into account, we expect the holomorphic $\bar{S}_{i} \rightarrow 0$ limit of the genus one B-model amplitude to be, up to an additive constant,

$$
F^{(1)}=\frac{1}{2} \log \left(\operatorname{det}\left(\frac{\partial z_{i}}{\partial S_{j}}\right) f(z)\right) .
$$

For the holomorphic ambiguity $f(z)$ we make the Ansatz $f(z)=\left(\prod_{i} \Delta_{i}^{\kappa_{i}}\right) I^{\kappa_{3}}$. The $\Delta_{i}$ are components of the discriminant of the Riemann surface. It splits into two factors, the conifold divisor $\Delta_{1}=z_{1} \cdot z_{2}$ and $\Delta_{2}=\left(x_{3}-x_{1}\right)\left(x_{3}-x_{2}\right)\left(x_{4}-x_{2}\right)\left(x_{4}-x_{1}\right)$. The exponents $\kappa_{i}$ determine the leading behavior of $F^{(1)}$ at the degeneration loci. At the conifold we expect [29] $\kappa_{1}=-\frac{1}{6}$. We fixed the $\kappa_{i}$ by comparing with any three coefficients of the matrix model computation, which we will present in sect. 4 , to $\kappa_{1}=-\frac{1}{6}, \kappa_{2}=\frac{2}{3}$ and $\kappa_{3}=-1$. Note that $I=0$ is not a discriminant component of the local geometry. The

3 Note that the sums in the expressions for the periods $S_{1}$ and $\Pi_{1}$ in appendix B of [19] should read $\sum_{n=1}^{\infty} c_{n} \Delta_{21}^{2 n} \frac{F^{2 n-2}(0)}{(2 n-2) !}$ and $\sum_{n=1}^{\infty} c_{n} \Delta_{21}^{2 n} \frac{G^{2 n-2}(0)}{(2 n-2) !}$, respectively. 
above ansatz for $f$ was motivated by simplicity. $I$ seems to appear in $f$ as a branch locus in the moduli space similarly as the $Z_{5}$ orbifold point $\Psi=0$ appears in the ambiguity of $F^{(1)}$ for the quintic in $[3] . F^{(1)}$ has the expansion

$$
\begin{aligned}
F^{(1)}= & -\frac{1}{12} \log \left(\frac{S_{1} S_{2}}{\Lambda^{6}}\right)+\frac{1}{6}\left(S_{1}-S_{2}\right)+\frac{g^{4}}{m^{6}}\left(\frac{7}{3} S_{1}^{2}-\frac{31}{3} S_{1} S_{2}+\frac{7}{3} S_{2}^{2}\right) \\
& +\frac{g^{6}}{m^{9}}\left(\frac{332}{9} S_{1}^{3}-\frac{923}{3} S_{1}^{2} S_{2}+\ldots\right) \\
& +\frac{g^{8}}{m^{12}}\left(\frac{1864}{3} S_{1}^{4}-\frac{47083}{6} S_{1}^{3} S_{2}+15349 S_{1}{ }^{2} S_{2}^{2}-\ldots\right) \\
& +\frac{g^{10}}{m^{15}}\left(\frac{54416}{5} S_{1}^{5}-187528 S_{1}^{4} S_{2}+570066 S_{1}^{3} S_{2}^{2}-\ldots\right) \\
& +\frac{g^{12}}{m^{18}}\left(\frac{1762048}{9} S_{1}^{6}-\frac{12980560 S_{1}}{3} S_{1}^{5} S_{2}+\frac{54863776}{3} S_{1}^{4} S_{2}^{2}-\frac{256344964}{9} S_{1}^{3} S_{2}^{3}+\ldots\right) \\
& +\mathcal{O}\left(S^{7}\right),
\end{aligned}
$$

where ... means antisymmetric completion.

\section{3. $\mathcal{F}^{(1)}$ from topological field theory}

In the decoupling limit (2.3), the amplitudes $\mathcal{F}^{(g)}$ give corrections to the low energy effective action of $\mathcal{N}=2$ super Yang-Mills theory of the form (2.6). In particular, $\mathcal{F}^{(1)}$ gives a term

$$
\int d^{4} x \mathcal{F}^{(1)}(a) \operatorname{Tr} R_{+}^{2}
$$

which can be integrated to obtain

$$
\frac{1}{2} \mathcal{F}^{(1)}(a)\left(\chi-\frac{3}{2} \sigma\right)
$$

Here $a$ is a coordinate in the moduli space of $\mathcal{N}=2$ super Yang-Mills, and $\chi$ and $\sigma$ denote the Euler characteristic and signature of the four-manifold, respectively. A coupling to gravity like (3.2) is natural when the theory is embedded in string theory, as we are doing here. Another way in which couplings to gravity are relevant is when the $\mathcal{N}=2$ theory is topologically twisted. Indeed, as shown in [6], the low-energy effective action of twisted $\mathcal{N}=2$ super Yang-Mills on an arbitrary four-manifold contains terms proportional to the Euler characteristic and the signature (these terms appear in any twisted gauge theory with $\mathcal{N}=2[8,9]$ or $\mathcal{N}=4$ [7] supersymmetry). As pointed out in [7],14], when the manifold is 
hyperKähler, the twisted and the physical theory agree, and we should therefore be able to compare the coupling (3.2) with the corresponding result in topological field theory. This was done in [14] for the $\mathcal{N}=4$ theory.

The geometry $I$ of the preceding section engineers $\mathcal{N}=2$ Yang-Mills theory with gauge group $S U(2)$ in the limit (2.3). On the other hand, the coupling to gravity that appears in the effective action of the twisted theory can be written [6,8] in terms of data of the Seiberg-Witten elliptic curve [4:50:

$$
-\frac{\chi}{2} \log \left(\frac{\Lambda d a}{d u}\right)+\frac{\sigma}{8} \log \left(\frac{\Delta}{\Lambda^{4}}\right),
$$

where $a(u)$ is the $a$-period of the Seiberg-Witten differential, $u$ the coordinate on the moduli space, and $\Delta$ is the discriminant of the curve. This should agree with (3.2) on a compact four-dimensional hyperKähler manifold. There are two such manifolds: $T^{4}$ and K3. For $T^{4}, \chi=\sigma=0$, and both (3.2) and (3.3) are trivial. For K3, $\chi=24, \sigma=-16$, and by comparing (3.2) to (3.3) we find:

$$
\mathcal{F}^{(1)}(a)=-\frac{1}{2} \log \left(\frac{\Lambda d a}{d u}\right)-\frac{1}{12} \log \left(\frac{\Delta}{\Lambda^{4}}\right) .
$$

Using the explicit expressions $\Delta=u^{2}-\Lambda^{4}$, and the explicit relation between $u$ and $a$ worked out in [24],

$$
u(a)=2 a^{2}\left(1+\frac{1}{2^{4}}\left(\frac{\Lambda}{a}\right)^{4}++\frac{5}{2^{12}}\left(\frac{\Lambda}{a}\right)^{8}+\frac{9}{2^{17}}\left(\frac{\Lambda}{a}\right)^{12}+\frac{1469}{2^{28}}\left(\frac{\Lambda}{a}\right)^{16}+\cdots\right),
$$

we computed the first few non-trivial corrections:

$$
\begin{aligned}
\mathcal{F}^{(1)}(a)=\frac{1}{6} \log \left(32 \frac{a}{\Lambda}\right)+\frac{1}{2^{13}}\left(\frac{\Lambda}{a}\right)^{8}+\frac{1}{3 \cdot 2^{14}}\left(\frac{\Lambda}{a}\right)^{12}+\frac{1647}{2^{29}}\left(\frac{\Lambda}{a}\right)^{16} \\
+\frac{981}{2^{31}}\left(\frac{\Lambda}{a}\right)^{20}+\frac{450137}{3 \cdot 2^{41}}\left(\frac{\Lambda}{a}\right)^{24}+\frac{45111}{2^{42}}\left(\frac{\Lambda}{a}\right)^{28}+\cdots
\end{aligned}
$$

This is precisely what we found in sect. 2 .

It is interesting to notice that, when $\mathcal{F}^{(1)}$ is written in terms of modular forms (by using for example the results in $[\mathbb{8}]$ ), one finds

$$
\mathcal{F}^{(1)}=-\log \eta(\tau)
$$

In this equation $\tau$ is the modular parameter of the Seiberg-Witten curve when written in the $\Gamma(2)$ description of [4], and it is related to the modular parameter $\tau_{0}$ of the $\Gamma^{0}(4)$ description of [5.24] by $\tau=\tau_{0} / 2$. Equation (3.7) is in agreement with the general considerations in [15] relating $F^{(1)}$ to $\eta(\tau)$ whenever the local Calabi-Yau geometry reduces to a Riemann surface. 


\section{Matrix model considerations}

The conjecture of Dijkgraaf and Vafa states that the cubic matrix model captures all the information about the $F^{(g)}$ of the $\widehat{I I}$ geometry. On the other hand, one can consider a limit that recovers the Seiberg-Witten exact solution to $S U(2), \mathcal{N}=2$ super Yang-Mills theory, together with its gravitational corrections [17,15, 16], which are given by the nonplanar sector of the matrix model. In this section we give some results for the cubic matrix model beyond the planar approximation. We compute the free energy up to sixth order in perturbation theory, providing in this way information up to $g=3$, and we use the loop equations to find an exact expression for $F^{(1)}$.

\subsection{Perturbative calculation}

The cubic matrix model has the potential

$$
W(\Phi)=\operatorname{tr}\left(\frac{m}{2} \Phi^{2}+\frac{g}{3} \Phi^{3}\right)=\sum_{i=1}^{N}\left(\frac{m}{2} \lambda_{i}^{2}+\frac{g}{3} \lambda_{i}^{3}\right)
$$

Classically the potential is extremized if the eigenvalues are in either one of the two critical points, i.e. at $a_{1}=0$ or $a_{2}=-m / g$. Quantum-mechanically the eigenvalues form two bands and there is eigenvalue tunneling between the two bands. We want to consider the metastable vacuum in which $N_{1}$ eigenvalues are at 0 and $N_{2}$ eigenvalues are at $-m / g$, with $N_{1}$ and $N_{2}$ fixed, subject only to the condition that $N_{1}+N_{2}=N$. This corresponds, in matrix model terminology, to a two-cut solution. This issue has already been addressed recently [16]. Here we will use a slightly different approach which avoids introducing ghost degrees of freedom. While the authors of [16] considered only the planar limit of the matrix model, we also compute non-planar contributions. While the former contain the information about the effective superpotential, the holomorphic couplings of the field theory to gravity are obtained from the non-planar part of the free energy.

The partition function $Z$ and free energy $F$ of the matrix model are:

$$
Z=e^{F}=\frac{1}{\operatorname{Vol}(U(N))} \int D \Phi \Phi e^{-W(\Phi)}=\frac{1}{N !(2 \pi)^{N}} \int \prod_{i} d \lambda_{i} \Delta^{2}(\lambda) \mathrm{e}^{-\frac{m}{2} \sum_{i} \lambda_{i}^{2}-\frac{g}{3} \sum_{i} \lambda_{i}^{3}}
$$


where $\Delta(\lambda)=\prod_{i<j}\left(\lambda_{i}-\lambda_{j}\right)$ is the Vandermonde determinant. We expand around the vacuum with $\lambda_{i}=0$ for $i=1, \ldots, N_{1}$ and $\lambda_{i}=-\frac{m}{g}$ for $i=N_{1}+1, \ldots, N$. Denoting the fluctuations by $\mu_{i}$ and $\nu_{j}$, the Vandermonde determinant becomes

$$
\Delta^{2}(\lambda)=\prod_{1 \leq i_{1}<i_{2} \leq N_{1}}\left(\mu_{i_{1}}-\mu_{i_{2}}\right)^{2} \prod_{1 \leq j_{1}<j_{2} \leq N_{2}}\left(\nu_{j_{1}}-\nu_{j_{2}}\right)^{2} \prod_{\substack{1 \leq i \leq N_{1} \\ 1 \leq j \leq N_{2}}}\left(\mu_{i}-\nu_{j}+\frac{m}{g}\right)^{2}
$$

We also expand the potential around this vacuum and get

$$
W=\sum_{i=1}^{N_{1}}\left(\frac{m}{2} \mu_{i}^{2}+\frac{g}{3} \mu_{i}^{3}\right)-\sum_{i=1}^{N_{2}}\left(\frac{m}{2} \nu_{i}^{2}-\frac{g}{3} \nu_{i}^{3}\right)+\frac{m^{3}}{6 g^{2}} N_{2}
$$

Notice that the propagator of the fluctuations around $-m / g$ has the 'wrong' sign. The interaction between the two sets of eigenvalues, which is given by the last factor in (4.3), can be exponentiated and included in the action, as in [18. This generates an interaction term between the two eigenvalue bands

$$
W_{\mathrm{int}}=-2 N_{1} N_{2} \log \frac{m}{g}+2 \sum_{k=1}^{\infty} \frac{1}{k}\left(\frac{g}{m}\right)^{k} \sum_{i, j} \sum_{p=0}^{k}(-1)^{p}\left(\begin{array}{l}
k \\
p
\end{array}\right) \mu_{i}^{p} \nu_{j}^{k-p}
$$

By rewriting the partition function in terms of matrices instead of their eigenvalues, we can represent this model as an effective two-matrix model, involving an $N_{1} \times N_{1}$ matrix $\Phi_{1}$, and an $N_{2} \times N_{2}$ matrix $\Phi_{2}$ :

$$
Z=\frac{1}{\operatorname{Vol}\left(U\left(N_{1}\right)\right) \times \operatorname{Vol}\left(U\left(N_{2}\right)\right)} \int D \Phi_{1} D \Phi_{2} \mathrm{e}^{-W_{1}\left(\Phi_{1}\right)-W_{2}\left(\Phi_{2}\right)-W\left(\Phi_{1}, \Phi_{2}\right)}
$$

where

$$
\begin{aligned}
W_{1}\left(\Phi_{1}\right) & =+\operatorname{tr}\left(\frac{1}{2} m \Phi_{1}^{2}+\frac{g}{3} \Phi_{1}^{3}\right) \\
W_{2}\left(\Phi_{2}\right) & =-\operatorname{tr}\left(\frac{1}{2} m \Phi_{2}^{2}-\frac{g}{3} \Phi_{2}^{3}\right) \\
W_{\text {int }}\left(\Phi_{1}, \Phi_{2}\right) & =2 \sum_{k=1}^{\infty} \frac{1}{k}\left(\frac{g}{m}\right)^{k} \sum_{p=0}^{k}(-1)^{p}\left(\begin{array}{c}
k \\
p
\end{array}\right) \operatorname{tr} \Phi_{1}^{p} \operatorname{tr} \Phi_{2}^{k-p} \\
& +N_{2} W\left(a_{2}\right)+N_{1} W\left(a_{1}\right)+2 N_{1} N_{2} \ln \left(\frac{m}{g}\right) .
\end{aligned}
$$

where $\operatorname{tr} \Phi_{1}^{0}=N_{1}, \operatorname{tr} \Phi_{2}^{0}=N_{2}, W\left(a_{1}\right)=0$ and $W\left(a_{2}\right)=\frac{m^{3}}{6 g^{2}}$. We have dropped the statistical factor $N ! /\left(N_{1} ! N_{2} !\right)$ which counts the number of ways to distribute the $N$ eigenvalues among the two critical points of the potential. This two-matrix model is (perturbatively) 
well defined if we choose $\Phi_{1}$ hermitian and $\Phi_{2}$ anti-hermitian, i.e. $\mu_{i}$ real and $\nu_{j}$ imaginary. We can now compute the free energy $F=\log (Z)$ is a straightforward way. $F$ consists of two parts, a perturbative part $F_{\text {pert }}=\log (Z(g) / Z(g=0))$ which vanishes for the free (Gaussian) model and a non-perturbative part $F_{\text {n.p. }}$ which gets contributions from the $U(N)$ group-volume factor. Both can be computed in a straightforward way. The perturbative part can be expanded as

$$
F_{\text {pert }}=-N_{1} W\left(a_{1}\right)-N_{2} W\left(a_{2}\right)+2 N_{1} N_{2} \ln \left(\frac{m}{g}\right)+\sum_{h=1}^{\infty} \sum_{\substack{g \geq 0 \\ h+2-2 g>0}}\left(\frac{g^{2}}{m^{3}}\right)^{h} F_{h, g}\left(N_{1}, N_{2}\right)
$$

where $F_{h, g}$ is a homogeneous polynomial in $N_{1}$ and $N_{2}$ of degree $h+2-2 g$. One finds, up to $h=6$,

$$
\begin{aligned}
& F_{\text {pert }}=- N_{1} W\left(a_{1}\right)-N_{2} W\left(a_{2}\right)+2 N_{1} N_{2} \ln \left(\frac{m}{g}\right) \\
&+\frac{g^{2}}{m^{3}}[\left.\left(\frac{2}{3} N_{1}^{3}-5 N_{1}^{2} N_{2}+5 N_{1} N_{2}^{2}-\frac{2}{3} N_{2}^{3}\right)+\frac{1}{6}\left(N_{1}-N_{2}\right)\right] \\
&+\frac{g^{4}}{m^{6}}\left[\left(\frac{8}{3} N_{1}^{4}-\frac{91}{3} N_{1}^{3} N_{2}+59 N_{1}^{2} N_{2}^{2}-\frac{91}{3} N_{1} N_{2}^{3}+\frac{8}{3} N_{2}^{4}\right)+\left(\frac{7}{3} N_{1}^{2}-\frac{31}{3} N_{1} N_{2}+\frac{7}{3} N_{2}^{2}\right)\right] \\
&+\frac{g^{6}}{m^{9}}\left[\left(\frac{56}{3} N_{1}^{5}-\frac{871}{3} N_{1}^{4} N_{2}+\frac{2636}{3} N_{1}^{3} N_{2}^{2}-\frac{2636}{3} N_{1}^{2} N_{2}^{3}+\frac{871}{3} N_{1} N_{2}^{4}-\frac{56}{3} N_{2}^{5}\right)\right. \\
&+\left.\left(\frac{332}{9} N_{1}^{3}-\frac{923}{3} N_{1}^{2} N_{2}+\frac{923}{3} N_{1} N_{2}^{2}-\frac{332}{9} N_{2}^{3}\right)+\frac{35}{6}\left(N_{1}-N_{2}\right)\right] \\
&+\frac{g^{8}}{m^{12}}[\left(\frac{512}{3} N_{1}^{6}-\frac{6823}{2} N_{1}^{5} N_{2}+\frac{28765}{2} N_{1}^{4} N_{2}^{2}-\frac{67310}{3} N_{1}^{3} N_{2}^{3} \pm \ldots\right) \\
&\left.+\left(\frac{1864}{3} N_{1}^{4}-\frac{47083}{6} N_{1}^{3} N_{2}+15349 N_{1}^{2} N_{2}^{2} \mp \ldots\right)+\left(338 N_{1}^{2}-1632 N_{1} N_{2}+338 N_{2}^{2}\right)\right] \\
&+\frac{g^{10}}{m^{15}}[ \frac{9152}{5}\left(N_{1}^{7}-45118 N_{1}^{6} N_{2}+247980 N_{1}^{5} N_{2}^{2}-540378 N_{1}^{4} N_{2}^{3} \pm \ldots\right) \\
&+\left(\frac{54416}{5} N_{1}^{5}-187528 N_{1}^{4} N_{2}+570066 N_{1}^{3} N_{2}^{2} \mp\right)+\left(\frac{66132}{5} N_{1}^{3}-120880 N_{1}^{2} N_{2} \mp \ldots\right) \\
&\left.+\frac{5005}{3}\left(N_{1}-N_{2}\right)\right] \\
&\left.+\left(\frac{1680704}{9}\left(N_{1}^{2}+N_{2}^{2}\right)-\frac{8748896}{9} N_{1} N_{2}\right)\right]+\ldots \\
&+\left(\frac{1762048}{9} N_{1}^{6}-\frac{12980560}{3} N_{1}^{5} N_{2}+\frac{54863776}{3} N_{1}^{4} N_{2}^{2}-\frac{256344964}{9} N_{1}^{3} N_{2}^{3} \pm \ldots\right) \\
&+\frac{g^{12}}{m^{18}}[\left(\frac{6536}{3} N_{1}^{8}-\frac{1933906}{3} N_{1}^{7} N_{2}+\frac{13258178}{3} N_{1}^{6} N_{2}^{2}-\frac{37761034}{3} N_{1}^{5} N_{2}^{3}+\frac{52780010}{3} N_{1}^{4} N_{2}^{4} \mp \ldots\right) \\
&\left(\frac{1305280}{3} N_{1}^{4}-\frac{18059582}{3} N_{1}^{3} N_{2}+11824166 N_{1}^{2} N_{2}^{2} \mp \ldots\right)
\end{aligned}
$$


where ... means again anti-symmetric completion. The non-perturbative contribution to the free energy is

$$
e^{F_{\mathrm{n} . \mathrm{p} .}}=m^{-\frac{1}{2}\left(N_{1}^{2}+N_{2}^{2}\right)}(2 \pi)^{-\frac{1}{2}\left(N_{1}+N_{2}\right)} \prod_{k=1}^{N_{1}} \Gamma(k) \prod_{l=1}^{N_{2}} \Gamma(l)
$$

With the help of the asymptotic expansion (see e.g. 30])

$$
\ln \left(\prod_{k=1}^{N} \Gamma(k)\right)=\frac{N^{2}}{2} \ln N-\frac{1}{12} \ln N-\frac{3}{4} N^{2}+\frac{1}{2} N \ln 2 \pi+\zeta^{\prime}(-1)+\sum_{g=2}^{\infty} \frac{B_{2 g}}{4 g(g-1)} \frac{1}{N^{2 g-2}}
$$

this becomes

$$
\begin{gathered}
F_{\text {n.p. }}=\frac{1}{2} N_{1}^{2} \ln \left(\frac{N_{1}}{m}\right)+\frac{1}{2} N_{2}^{2} \ln \left(\frac{N_{2}}{m}\right)-\frac{3}{4}\left(N_{1}^{2}+N_{2}^{2}\right)-\frac{1}{12} \ln \left(N_{1} N_{2}\right) \\
+2 \zeta^{\prime}(-1)+\sum_{g=2}^{\infty} \frac{B_{2 g}}{4 g(g-1)}\left(\frac{1}{N_{1}^{2 g-2}}+\frac{1}{N_{2}^{2 g-2}}\right)
\end{gathered}
$$

Comparing with the prepotential of the gauge theory with cubic superpotential, which can be extracted from the results given in [19], we find it to be in precise agreement with the leading terms, at each order in the coupling constant, of the free energy computed from the matrix model if we identify $N_{i} \rightarrow S_{i}$. The only part of the gauge theory result which is not directly determined by the matrix model is the dependence on the cut-off $\Lambda$.

\section{2. $F^{(1)}$ from the loop equations}

The expression for the free energy (4.9) was obtained by doing perturbation theory. However one can obtain analytic expressions for the genus $g$ free energies by using the loop equations of the matrix model. These equations were used systematically in [31] in the one-cut case, and extended to the multi-cut case in [32]. Before moving on to the comparison with topological string theory calculations, we derive an analytic expression for $F^{(1)}$ for the cubic matrix model which can be easily expanded to high powers in $N_{1}$ and $N_{2}$. The computation closely parallels the derivation in 32] of the two-cut solution, to which we refer for further details. The reason why we cannot directly adopt the result of [32] is that instead of imposing the absence of eigenvalue tunneling between the two bands (equal chemical potential), we fix $N_{1}$ and $N_{2}$, i.e. we impose

$$
\int_{x_{3}}^{x_{4}} \rho(x) d x=N_{1}, \quad \text { and } \quad \int_{x_{1}}^{x_{2}} \rho(x) d x=N_{2} .
$$


instead of $\int_{x_{2}}^{x_{3}} \rho(x) d x=0$. In other words, we are not looking for the true vacuum but consider a meta-stable vacuum with fixed $N_{1}$ and $N_{2}$ 国. Here $\rho(x)$ is the eigenvalue density which is given by the discontinuity of the resolvent of the matrix $\Phi$. For the cubic superpotential $W(x)=\frac{m}{2} x^{2}+\frac{g}{3} x^{3}$ it is $\rho(x)=\frac{g}{2 \pi i} \sqrt{\prod_{i=1}^{4}\left(x-x_{i}\right)}=\frac{1}{2 \pi i} \sqrt{W^{\prime 2}(x)-f_{1}(x)}$ where $f_{1}$ is a polynomial of order one whose two coefficients parameterize the widths of the two branch cuts. Note that $N_{i}$ are given by the same integrals as $S_{i}$ in the gauge theory. The two conditions (4.13) are not independent since $N_{1}+N_{2}=N$. The other conditions, which follow from the asymptotic behavior of the resolvent, are exactly as in [32]. Following the steps in [32] one finds (we give a few intermediate results of the computation in appendix B)

$F^{(1)}=-\frac{1}{24} \sum_{i=1}^{4} \ln M_{i}-\frac{1}{2} \ln K(k)-\frac{1}{12} \sum_{i<j} \ln \left(x_{i}-x_{j}\right)^{2}+\frac{1}{8} \ln \left(x_{1}-x_{3}\right)^{2}+\frac{1}{8} \ln \left(x_{2}-x_{4}\right)^{2}+$ const.

where $K(k)$ is a complete elliptic integral with modulus

$$
k^{2}=\frac{\left(x_{1}-x_{2}\right)\left(x_{3}-x_{4}\right)}{\left(x_{1}-x_{3}\right)\left(x_{2}-x_{4}\right)}
$$

and the moments $M_{i}$ are defined as

$$
M_{i}=\oint_{\mathcal{C}} \frac{d x}{2 \pi i} \frac{W^{\prime}(x)}{\left(x-x_{i}\right) \sqrt{\prod_{i=1}^{4}\left(x-x_{i}\right)}}
$$

where the contour $\mathcal{C}$ encloses both cuts. For cubic $W, M_{i}=g$ for all $i=1, \ldots, 4$, as one shows by deforming $\mathcal{C}$ such as to enclose infinity.

It remains to express $F^{(1)}$ as a series in $N_{1}$ and $N_{2}$, with coefficients depending on $m$ and $g$, the two parameters of $W$. For this purpose it is convenient to change variables, as in [19], $\left(x_{1}, x_{2}, x_{3}, x_{4}\right) \rightarrow\left(\Delta_{21}, \Delta_{43}, Q, I\right)$, where the explicit relations were given in (2.18).

4 The complete matrix-model partition function involves a sum over all possible eigenvalue distributions and does not have a topological expansion, as explained in [33]. This subtlety is, however, not relevant here as we fix $N_{1}$ and $N_{2}$. 
Inserting (2.19) with $S_{i}$ replaced by $N_{i}$, we obtain

$$
\begin{aligned}
F^{(1)} & =-\frac{1}{12} \log \left(N_{1} N_{2}\right)+\frac{g^{2}}{m^{3}}\left(\frac{N_{1}}{6}-\frac{N_{2}}{6}\right)+\frac{g^{4}}{m^{6}}\left(\frac{7 N_{1}^{2}}{3}-\frac{31 N_{1} N_{2}}{3}+\frac{7 N_{2}^{2}}{3}\right) \\
& +\frac{g^{6}}{m^{9}}\left(\frac{332 N_{1}^{3}}{9}-\frac{923 N_{1}^{2} N_{2}}{3} \pm \ldots\right)+\frac{g^{8}}{m^{12}}\left(\frac{1864 N_{1}^{4}}{3}-\frac{47083 N_{1}^{3} N_{2}}{6}+15349 N_{1}^{2} N_{2}^{2} \mp \ldots\right) \\
& +\frac{g^{10}}{m^{15}}\left(\frac{54416 N_{1}^{5}}{5}-187528 N_{1}^{4} N_{2}+570066 N_{1}^{3} N_{2}^{2} \mp \ldots\right) \\
& +\frac{g^{12}}{m^{18}}\left(\frac{1762048 N_{1}{ }^{6}}{9}-\frac{12980560 N_{1}^{5} N_{2}}{3}+\frac{54863776 N_{1}^{4} N_{2}^{2}}{3}-\frac{256344964 N_{1}{ }^{3} N_{2}{ }^{3}}{9} \pm \ldots\right) \\
& +\mathcal{O}\left(N^{7}\right)
\end{aligned}
$$

where ... means, as before, antisymmetric completion. This expression agrees exactly with the expansion of $F^{(1)}$ for the local geometry II 2.21) that we obtained in section 2 , with the identification $S_{i}=N_{i}, i=1,2$. This provides a one-loop test of the relation conjectured in [13].

Using the iterative procedure developed in [31], one can, with some effort, derive expressions for the higher genus contributions to the free energy.

\section{3. $\mathcal{N}=2$ Yang-Mills from the matrix model}

As explained in 17] 15], 16], using the results of the cubic matrix model one can derive results for $S U(2), \mathcal{N}=2$ Yang-Mills theory. The idea is the following: the cubic matrix model corresponds to the $\mathcal{N}=2$ theory broken down to $\mathcal{N}=1$ by adding the tree level superpotential $W(\Phi)$ for the chiral superfield $\Phi$. By taking $g, m \rightarrow 0$ and keeping $\Delta$ fixed, we recover the pure $\mathcal{N}=2$ theory. In general, a tree level superpotential of order $r+1$ allows one to recover the moduli space of the $S U(r)$ theory. In our case, we can recover the $S U(2)$ theory, and $\Delta$ will then be related to the well-known $u$-modulus of Seiberg-Witten theory [4] [5]. Some results for the prepotential for general $r$ have been recently obtained in 34 .

In order to recover the $\mathcal{N}=2$ results, we first have to find the relation between the matrix model variables and the usual variables of the Seiberg-Witten solution. Following [16], we extremize the effective superpotential of the gauge theory $W_{\text {eff }}=\frac{\partial F^{(0)}}{\partial S_{1}}+\frac{\partial F^{(0)}}{\partial S_{2}}$ 
where (cf. (4.9) and (4.12) with $N_{i} \rightarrow S_{i}$ and the $\Lambda$ dependence added on dimensional grounds)

$$
\begin{gathered}
F^{(0)}=\frac{1}{2} S_{1}^{2} \log \left(\frac{S_{1}}{m \Lambda^{2}}\right)+\frac{1}{2} S_{2}^{2} \log \left(\frac{S_{2}}{m \Lambda^{2}}\right)-\frac{3}{4}\left(S_{1}^{2}+S_{2}^{2}\right)+2 S_{1} S_{2} \log \left(\frac{m}{\Lambda g}\right) \\
+\frac{1}{g \Delta^{3}}\left(\frac{2}{3} S_{1}^{3}-5 S_{1}^{2} S_{2}+5 S_{1} S_{2}^{2}-\frac{2}{3} S_{2}^{3}\right)+\mathcal{O}\left(S^{4}\right)
\end{gathered}
$$

Here we have ignored terms $\mathcal{O}(\mathcal{S})$ since they will not contribute to the positions of the extrema of $W_{\text {eff }}$. Extremizing $W_{\text {eff }}$, i.e. solving $\partial_{S_{i}} W_{\text {eff }}=0$, requires $S_{1}=-S_{2} \equiv S$ with

$$
\frac{S}{g \Delta^{3}}=\left(\frac{\Lambda}{\Delta}\right)^{4}\left(1+6\left(\frac{\Lambda}{\Delta}\right)^{4}+140\left(\frac{\Lambda}{\Delta}\right)^{8}+4620\left(\frac{\Lambda}{\Delta}\right)^{12}+180180\left(\frac{\Lambda}{\Delta}\right)^{16}+7759752\left(\frac{\Lambda}{\Delta}\right)^{20}+\cdots\right)
$$

as obtained in [16]. Note that the relation $S_{1}+S_{2}=0$ implies that $f_{1}$ in (2.2) vanishes. This, on the other hand means that the contours which define $S_{1}$ and $S_{2}$ can be deformed into each other without picking up a contribution from the point at infinity. In other words, we can add this point and consider a compact Riemann surface, as in SeibergWitten theory. However, the scale $\Lambda$ which appears in (4.19) is, a priori, not identical with the scale appearing in Seiberg-Witten theory. To find their relation, we relate the curve $y^{2}=W^{\prime}(x)^{2}+f_{0}=g^{2} \prod\left(x-x_{i}\right)$ to the Seiberg-Witten curve. First we shift $x \rightarrow x-\frac{1}{2} \Delta$. Using the relations (2.18) and the solutions $\Delta_{21}(S,-S)$ and $\Delta_{43}(S,-S)$ (c.f. (2.19) one finds, after rescaling $y \rightarrow g y, y^{2}=\left(x^{2}-\frac{1}{4} \Delta^{2}\right)^{2}-4 \Lambda^{4}$. Comparing this with the $S U(2)$ SW curve, $y^{2}=\left(x^{2}-u\right)^{2}-\Lambda_{\mathrm{SW}}^{4}$ leads to the identifications

$$
u=\frac{1}{4} \Delta^{2}, \quad \text { and } \quad \Lambda=\frac{1}{\sqrt{2}} \Lambda_{\mathrm{SW}}
$$

This, together with (4.19), gives the relation between the variables in the matrix model computation and the usual variables in Seiberg-Witten theory.

Since in order to obtain pure $S U(2)$ super Yang-Mills we take a limit of the matrix model, in order to compare the results of the matrix model calculation with the results obtained in sections 2 and 3 we have to be very careful and look for quantities that do not vanish or diverge as $g \rightarrow 0$. For example, $S$ vanishes as $g \rightarrow 0$, while $S / g$ is independent of $g$ and can be expressed solely in terms of $\Delta$, as it is apparent from (4.19). When we express $F^{(r)}$ in terms of $S / g$, we see that it is given by $g^{2-2 r}$, times a function of $S / g$. Therefore, if $r \neq 1$, the resulting quantity depends on $g$ and vanishes or diverges as $g \rightarrow 0$. This is related to the fact that $F^{(r)}$ is not a function in moduli space, but rather a section of a line bundle $\mathcal{L}^{2-2 r}$, i.e. for $r \neq 1$ it is not invariant under the gauge transformations of special 
geometry. This also indicates that, in order to compare the matrix model calculations with the results of section 2 and 3, we should focus on gauge-invariant quantities.

Following this idea, in the case of $F^{(0)}$ it is clear that the appropriate quantities are the second derivatives w.r.t. the moduli, i.e. the $\tau_{i j}$ couplings, which are independent of $g$ [17]. One can check [16] that the $\tau_{i j}$ computed in the matrix model lead to the right result for $\tau$ in the $\mathcal{N}=2$ theory. Since $F^{(1)}$ is gauge-invariant and can be expressed in terms of $\Delta$, with no $g$ dependence, one should be able to compare directly the $F^{(1)}$ of (3.6) with the $F^{(1)}$ obtained in the matrix model. Indeed, using (4.19), 4.20), and the relation between the $u$ modulus and the $a$ variable given in (3.5), we find that the $F^{(1)}$ of $(4.17)$ reproduces (3.6) after evaluating it at the extremum . It seems less straightforward to relate the matrix model results for $F^{(g)}$ for $g>1$ to the couplings $\mathcal{F}^{(g)}$ in Seiberg-Witten theory derived from string theory. In the final section we will, however, find agreement between the expressions (2.17) and a recent conjecture by Nekrasov.

\section{Comparison with instanton computations}

The semiclassical expansion of the $\mathcal{N}=2$ prepotential of Yang-Mills theory can be obtained by direct computation of instanton corrections. In a recent tour de force Nekrasov [10] (see also [11]) was able to provide general expressions for the $n$-th instanton contribution to the $\mathcal{N}=2, S U(N)$ prepotential, with or without matter. The answer for the $n$-th instanton correction has the form $\mathcal{F}_{n}\left(a, \epsilon_{1}, \epsilon_{2}\right)$ and it is an analytic function in $\epsilon_{1,2}$. For $\epsilon_{1}=-\epsilon_{2}=\epsilon$, one can expand this as

$$
\epsilon^{-2} \mathcal{F}_{n}(a, \epsilon)=\sum_{k=0}^{\infty} \mathcal{F}_{n}^{(g)}(a) \epsilon^{2 g-2}
$$

The first coefficient in this expansion, $\mathcal{F}_{n}^{(0)}(a)$, gives the prepotential of the corresponding $\mathcal{N}=2$ theory. It was conjectured in [10] that the remaining coefficients $\mathcal{F}_{n}^{(g)}(a), g \geq 1$, give the $n$-instanton correction to the gravitational couplings $\mathcal{F}^{(g)}$ of the $\mathcal{N}=2$ theory. Here we provide nontrivial evidence for this conjecture by comparing some of the instanton computations of [10,11] with the results of section 2 (and, therefore, with the matrix model computations).

\footnotetext{
5 Note that the scale appearing in sect. 3 is, in fact, $\Lambda_{\mathrm{SW}}$.
} 
In [11] $\mathcal{F}_{n}\left(\epsilon_{1}, \epsilon_{2}\right)$ was explicitly computed for $S U(2)$, up to $n=4$. To compare with our results in section 2, we restore units as follows: $\mathcal{F}_{n} \rightarrow(\Lambda / 2)^{4 n} \mathcal{F}_{n}$. For $n=1$ one finds

$$
\epsilon^{-2} \mathcal{F}_{1}(\epsilon)=\frac{\epsilon^{-2}}{2^{5}} \frac{\Lambda^{4}}{a^{2}}
$$

This says that the one-instanton contribution to $\mathcal{F}^{(g)}$ vanishes for $g>0$, which agrees with (2.16) and (2.17). For $n=2$, one finds:

$$
\begin{aligned}
\epsilon^{-2} \mathcal{F}_{2}(\epsilon) & =\frac{\Lambda^{8}}{2^{8}} \frac{10 a^{2}-\epsilon^{2}}{8 \epsilon^{2} a^{4}\left(4 a^{2}-\epsilon^{2}\right)^{2}} \\
& =\epsilon^{-2} \frac{5}{2^{14}} \frac{\Lambda^{8}}{a^{6}}+\frac{1}{2^{13}} \frac{\Lambda^{8}}{a^{8}}+\epsilon^{2} \frac{11}{2^{18}} \frac{\Lambda^{8}}{a^{10}}+\epsilon^{4} \frac{7}{2^{19}} \frac{\Lambda^{8}}{a^{12}}+\cdots
\end{aligned}
$$

For $n=3$, one finds from [11]:

$$
\begin{aligned}
\epsilon^{-2} \mathcal{F}_{3}(\epsilon) & =\frac{\Lambda^{12}}{2^{12}} \frac{18 a^{4}-13 a^{2} \epsilon^{2}+\epsilon^{4}}{24 \epsilon^{2} a^{6}\left(a^{2}-\epsilon^{2}\right)^{2}\left(4 a^{2}-\epsilon^{2}\right)^{2}} \\
& =\epsilon^{-2} \frac{3}{2^{18}} \frac{\Lambda^{12}}{a^{10}}+\frac{1}{3 \cdot 2^{14}} \frac{\Lambda^{12}}{a^{12}}+\epsilon^{2} \frac{117}{2^{22}} \frac{\Lambda^{12}}{a^{14}}+\epsilon^{4} \frac{293}{2^{23}} \frac{\Lambda^{12}}{a^{16}}+\cdots
\end{aligned}
$$

Finally, for $n=4$, one can extract from [11]:

$$
\begin{aligned}
\epsilon^{-2} \mathcal{F}_{4}(\epsilon)= & \frac{\Lambda^{16}}{2^{16}} \frac{23504 a^{10}-70872 a^{8} \epsilon^{2}+67461 a^{6} \epsilon^{4}-26339 a^{4} \epsilon^{6}+3708 a^{2} \epsilon^{8}-162 \epsilon^{10}}{128 \epsilon^{2} a^{8}\left(4 a^{2}-9 \epsilon^{2}\right)^{2}\left(a^{2}-\epsilon^{2}\right)^{2}\left(4 a^{2}-\epsilon^{2}\right)^{4}} \\
& =\epsilon^{-2} \frac{1469}{2^{31}} \frac{\Lambda^{16}}{a^{14}}+\frac{1647}{2^{29}} \frac{\Lambda^{16}}{a^{16}}+\epsilon^{2} \frac{171201}{2^{34}} \frac{\Lambda^{16}}{a^{18}}+\epsilon^{4} \frac{985823}{2^{35}} \frac{\Lambda^{16}}{a^{20}}+\ldots
\end{aligned}
$$

We see that, after relabelling $\epsilon \rightarrow i \epsilon$, the coefficients in the above expansions are in perfect agreement with the results in (2.14), (2.16) and (2.17).

Acknowledgements: We thank R. Flume, S. Gukov, V. Kazakov, I. Kostov, C. Kristjansen and C. Vafa for valuable discussions. The work of M.M. was supported by the grant NSF-PHY/98-02709; A.K. and S.T. would like to thank the Erwin-Schrödinger-Institut in Vienna, where part of this work was done, for hospitality. They were also partially supported by the European Commission TMR programme ERBFMRX-CT96-0045 and the work of S.T. in addition by GIF, the German-Israeli Foundation for Scientific Research. 


\section{Appendix A.}

We collect the first few polynomials $g_{n}^{(g)}$ which enter the topological amplitudes (2.12).

$$
\begin{aligned}
& h_{1}^{(2)}=(1-q)^{4} \\
& h_{2}^{(2)}=\frac{1}{2}\left(2-17 q+80 q^{2}+1190 q^{3}+80 q^{4}-17 q^{5}+2 q^{6}\right) \\
& h_{3}^{(2)}=\frac{1}{3}\left(3-38 q+1712 q^{2}+48326 q^{3}+124634 q^{4}+\ldots\right) \\
& h_{4}^{(2)}=\frac{1}{4}\left(4-67 q+14592 q^{2}+711059 q^{3}+4924138 q^{4}+9244668 q^{5}+\ldots\right) \\
& h_{5}^{(2)}=\frac{1}{5}\left(5-104 q+76705 q^{2}+6090098 q^{3}+82568187 q^{4}+358139062 q^{5}+580752958 q^{6}+\ldots\right)
\end{aligned}
$$

$$
\begin{aligned}
h_{1}^{(3)}= & (1-q)^{6} \\
h_{2}^{(3)}= & \frac{1}{8}\left(1+4 q+q^{2}\right)\left(8-125 q+1016 q^{2}-8854 q^{3}+1016 q^{4}-125 q+8 q^{6}\right) \\
h_{3}^{(3)}= & \frac{1}{3^{3}}\left(3^{3}-428 q+207 q^{2}-584608 q^{3}-6606954 q^{4}-13969512 q^{5}-\ldots\right) \\
h_{4}^{(3)}= & \frac{1}{4^{3}}\left(4^{3}-1275 q-62364 q^{2}-14760656 q^{3}-289184988 q^{4}-1451906781 q^{5}-2450425504 q^{6}-\ldots\right) \\
h_{5}^{(3)}= & \frac{1}{5^{3}}\left(5^{3}-2994 q-655802 q^{2}-183946424 q^{3}-5822909304 q^{4}-53583541710 q^{5}\right. \\
& \left.\quad-190175648587 q^{6}-288031344528 q^{7}-\ldots\right)
\end{aligned}
$$

They were used to derive (2.17). Note that the $h_{d_{1}}^{(g)}$ can be used to write down the conjecturally integer Gopakumar-Vafa invariants for $\mathcal{O}(-2,-2) \rightarrow \mathbb{F}_{0}$ (see e.g. [26]) $n_{d_{1}, d_{2}}^{(g)}$ for $g<4, d_{1}<6$ and $d_{2}$ arbitrary, e.g.

$$
\left\{n_{4, d_{2}}^{(3)}, d_{2}=0, \ldots\right\}=\{0,0,15,4680,184056,3288688,36882969,300668486 \text {, }
$$

$$
1935031484,10359890196,47820549652,195274337280,719145083800, \ldots\}
$$

We checked integrality of the $n_{d_{1}, d_{2}}^{(g)}$ for $g<4, d_{1}<6$ and $d_{2} \leq 2000$.

\section{Appendix B.}

We collect some of the intermediate results which are needed to derive (4.14). As we mentioned in sect. 4 , the derivation is almost identical to that in sect. 6 of [32]. Instead of 
the elliptic integrals $K_{i}$ defined there, we encounter (we choose $x_{1}<x_{2}<x_{3}<x_{4}$ )

$$
K_{i}=\int_{x_{3}}^{x_{4}} \frac{\sqrt{\left(x-x_{1}\right)\left(x-x_{2}\right)\left(x-x_{3}\right)\left(x-x_{4}\right)}}{\left(x-x_{i}\right)} d x
$$

They arise from varying the constraint that $N_{1}=\int_{x_{3}}^{x_{4}} \rho(x) d x$ is kept fixed rather than requiring $\int_{x_{2}}^{x_{3}} \rho(x) d x=0$ as in [32]. Explicit calculation gives [35]

$$
\begin{gathered}
K_{1}=i X\left\{\left(-\alpha^{6}+2 \alpha^{4}+\alpha^{2} k^{2}-2 \alpha^{4} k^{2}\right) E(k)-\left(k^{2}-\alpha^{2}\right)\left(\alpha^{4}-2 \alpha^{2}+k^{2}\right) K(k)\right. \\
\left.+\left(\alpha^{8}-4 \alpha^{6} k^{2}+6 \alpha^{4} k^{2}-4 \alpha^{2} k^{2}+k^{4}\right) \Pi\left(\alpha^{2}, k^{2}\right)\right\} \\
K_{2}=i X\{- \\
\quad \alpha^{2}\left(\alpha^{4}-2 \alpha^{2}-2 \alpha^{2} k^{2}+3 k^{2}\right) E(k)-\left(k^{2}-\alpha^{2}\right)\left(\alpha^{4}-2 \alpha^{2}+4 \alpha^{2} k^{2}-3 k^{2}\right) K(k) \\
\left.\quad+\left(\alpha^{8}-6 \alpha^{4} k^{2}+4 \alpha^{2} k^{4}+4 \alpha^{2} k^{2}-3 k^{4}\right) \Pi\left(\alpha^{2}, k^{2}\right)\right\} \\
K_{3}=i X\left\{\left(3 \alpha^{6}-2 \alpha^{4}-2 \alpha^{4} k^{2}+\alpha^{2} k^{2}\right) E(k)+\left(k^{2}-\alpha^{2}\right)\left(3 \alpha^{4}-2 \alpha^{2}-k^{2}\right) K(k)\right. \\
\left.\quad+\left(-3 \alpha^{8}+4 \alpha^{6}+4 \alpha^{6} k^{2}-6 \alpha^{4} k^{2}+k^{4}\right) \Pi\left(\alpha^{2}, k\right)\right\} \\
K_{4}=i X\left\{\left(-\alpha^{6}-2 \alpha^{4}+2 \alpha^{4} k^{2}+\alpha^{2} k^{2}\right) E(k)-\left(k^{2}-\alpha^{2}\right)\left(\alpha^{4}+2 \alpha^{2}-4 \alpha^{2} k^{2}+k^{2}\right) K(k)\right. \\
\left.\quad+\left(\alpha^{8}-4 \alpha^{6}+6 \alpha^{4} k^{2}-4 \alpha^{2} k^{4}+k^{4}\right) \Pi\left(\alpha^{2}, k\right)\right\}
\end{gathered}
$$

where

$$
\begin{aligned}
\alpha^{2} & =\frac{\left(x_{4}-x_{3}\right)}{\left(x_{4}-x_{2}\right)} \\
k^{2} & =\frac{\left(x_{1}-x_{2}\right)\left(x_{3}-x_{4}\right)}{\left(x_{1}-x_{3}\right)\left(x_{2}-x_{4}\right)} \\
X & =\frac{1}{4} \frac{\left(x_{1}-x_{3}\right)^{3 / 2}\left(x_{2}-x_{4}\right)^{7 / 2}}{\left(x_{4}-x_{3}\right)^{2}\left(x_{3}-x_{2}\right)}
\end{aligned}
$$

Next we have to solve eqs. (6.1) in [32] for the coefficients $\alpha_{i}$, however with $K_{i}$ as given above. We find

$$
\begin{aligned}
& \alpha_{1}=\frac{1}{\left(x_{1}-x_{2}\right)}\left[1-\frac{\left(x_{4}-x_{2}\right)}{\left(x_{4}-x_{1}\right)} \frac{E(k)}{K(k)}\right] \\
& \alpha_{2}=\frac{1}{\left(x_{2}-x_{1}\right)}\left[1-\frac{\left(x_{3}-x_{1}\right)}{\left(x_{3}-x_{2}\right)} \frac{E(k)}{K(k)}\right] \\
& \alpha_{3}=\frac{1}{\left(x_{3}-x_{4}\right)}\left[1-\frac{\left(x_{4}-x_{2}\right)}{\left(x_{3}-x_{2}\right)} \frac{E(k)}{K(k)}\right] \\
& \alpha_{4}=\frac{1}{\left(x_{4}-x_{3}\right)}\left[1-\frac{\left(x_{3}-x_{1}\right)}{\left(x_{4}-x_{1}\right)} \frac{E(k)}{K(k)}\right]
\end{aligned}
$$

With these values for $k^{2}$ and $\alpha_{i}$, the derivation of $F^{(1)}$ proceeds exactly as in 32] and finally leads to (4.14). 


\section{References}

[1] S. Katz, A. Klemm and C. Vafa, "Geometric engineering of quantum field theories," Nucl. Phys. B 497, 173 (1997); hep-th/9609239.

[2] S. Katz, P. Mayr and C. Vafa, "Mirror symmetry and exact solution of 4D N = 2 gauge theories. I," Adv. Theor. Math. Phys. 1, 53 (1998) arXiv:hep-th/9706110.

[3] M. Bershadsky, S. Cecotti, H. Ooguri and C. Vafa, "Kodaira-Spencer theory of gravity and exact results for quantum string amplitudes," Commun. Math. Phys. 165, 311 (1994), hep-th/9309140.

[4] N. Seiberg and E. Witten, "Electric - magnetic duality, monopole condensation, and confinement in $\mathcal{N}=2$ supersymmetric Yang-Mills theory," Nucl. Phys. B 426, 19 (1994), Erratum-ibid. B 430, 485 (1994), hep-th/9407087.

[5] N. Seiberg and E. Witten, "Monopoles, duality and chiral symmetry breaking in $\mathcal{N}=2$ supersymmetric QCD," Nucl. Phys. B 431, 484 (1994), hep-th/9408099.

[6] E. Witten, "On $S$ duality in Abelian gauge theory," hep-th/9505186.

[7] C. Vafa and E. Witten, "A strong coupling test of $S$ duality," Nucl. Phys. B 431, 3 (1994), hep-th/9408074.

[8] G. Moore and E. Witten, "Integration over the $u$-plane in Donaldson theory," Adv. Theor. Math. Phys. 1, 298 (1998), hep-th/9709193.

[9] A. Losev, N. Nekrasov and S. L. Shatashvili, "Issues in topological gauge theory," Nucl. Phys. B 534, 549 (1998), hep-th/9711108. M. Mariño and G. Moore, "The DonaldsonWitten function for gauge groups of rank larger than one," Commun. Math. Phys. 199, 25 (1998), hep-th/9802185.

[10] N. A. Nekrasov, "Seiberg-Witten prepotential from instanton counting," hep-th/0206161.

[11] R. Flume and R. Poghossian, "An algorithm for the microscopic evaluation of the coefficients of the Seiberg-Witten prepotential," hep-th/0208176.

[12] C. Vafa, "Superstrings and topological strings at large $N$," J. Math. Phys. 42, 2798 (2001), hep-th/0008142.

[13] R. Dijkgraaf and C. Vafa, "Matrix models, topological strings, and supersymmetric gauge theories," Nucl. Phys. B 644, 3 (2002), hep-th/0206255.

[14] R. Dijkgraaf and C. Vafa, "On geometry and matrix models," Nucl. Phys. B 644, 21 (2002), hep-th/0207106.

[15] R. Dijkgraaf and C. Vafa, "A perturbative window into non-perturbative physics," hep-th/0208048. 
[16] R. Dijkgraaf, S. Gukov, V. A. Kazakov and C. Vafa, "Perturbative analysis of gauged matrix models," hep-th/0210238.

[17] F. Cachazo and C. Vafa, "NN=1 and $\mathcal{N}=2$ geometry from fluxes," hep-th/0206017.

[18] M. Aganagic, A. Klemm, M. Mariño and C. Vafa, "Matrix model as a mirror of Chern-Simons theory," hep-th/0211098.

[19] F. Cachazo, K. A. Intriligator and C. Vafa, "A large $N$ duality via a geometric transition," Nucl. Phys. B 603, 3 (2001), hep-th/0103067.

[20] S. Kachru, S. Katz, A. E. Lawrence and J. McGreevy, "Open string instantons and superpotentials," Phys. Rev. D 62, 026001 (2000), hep-th/9912151.

[21] S. Kachru, A. Klemm, W. Lerche, P. Mayr and C. Vafa, "Nonperturbative results on the point particle limit of $\mathcal{N}=2$ heterotic string compactifications," Nucl. Phys. B 459, 537 (1996), hep-th/9508155.

[22] T. M. Chiang, A. Klemm, S. T. Yau and E. Zaslow, 'Local mirror symmetry: Calculations and interpretations," Adv. Theor. Math. Phys. 3, 495 (1999), hep-th/9903053.

[23] M. Bershadsky, S. Cecotti, H. Ooguri and C. Vafa, "Holomorphic anomalies in topological field theories," Nucl. Phys. B 405, 279 (1993) hep-th/9302103.

[24] A. Klemm, W. Lerche and S. Theisen, "Nonperturbative effective actions of $\mathcal{N}=2$ supersymmetric gauge theories," Int. J. Mod. Phys. A 11, 1929 (1996), hep-th/9505150.

[25] A. Klemm and E. Zaslow, "Local mirror symmetry at higher genus," hep-th/9906046.

[26] S. Katz, A. Klemm and C. Vafa, "M-theory, topological strings and spinning black holes," Adv. Theor. Math. Phys. 3, 1445 (1999), hep-th/9910181.

[27] S. Hosono, "Counting BPS states via holomorphic anomaly equations," hep-th/0206206.

[28] M. Aganagic, M. Mariño and C. Vafa, "All loop topological string amplitudes from Chern-Simons theory," hep-th/0206164.

[29] C. Vafa, "A stringy test of the fate of the conifold," Nucl. Phys. B 447, 252 (1995), hep-th/9505023.

[30] H. Ooguri and C. Vafa, "Worldsheet derivation of a large $N$ duality," Nucl. Phys. B 641, 3 (2002), hep-th/0205297.

[31] J. Ambjorn, L. Chekhov, C. F. Kristjansen and Y. Makeenko, "Matrix model calculations beyond the spherical limit," Nucl. Phys. B 404, 127 (1993), Erratum-ibid. B 449, 681 (1995), hep-th/9302014.

[32] G. Akemann, "Higher genus correlators for the Hermitian matrix model with multiple cuts," Nucl. Phys. B 482, 403 (1996), hep-th/9606004. 
[33] G. Bonnet, F. David and B. Eynard, "Breakdown of universality in multi-cut matrix models," J. Phys. A 33, 6739 (2000), cond-mat/0003324.

[34] S. Naculich, H. Schnitzer and N. Wyllard, "The $\mathcal{N}=2 U(N)$ gauge theory prepotential and periods from a perturbative matrix model calculation," hep-th/0211123.

[35] P.F. Byrd and M.D. Friedman, Handbook of Elliptic Integrals for Engineers and Scientists, Springer Verlag, New York 1971. 